\title{
Parameterized Hilbert-Type Integral Inequalities in the Whole Plane
}

\author{
Qiliang Huang, ${ }^{1}$ Shanhe $\mathrm{Wu}^{2}$ and Bicheng Yang ${ }^{1}$ \\ ${ }^{1}$ Department of Mathematics, Guangdong University of Education, Guangzhou, Guangdong 510303, China \\ ${ }^{2}$ Department of Mathematics and Computer Science, Longyan University, Longyan, Fujian 364012, China
}

Correspondence should be addressed to Shanhe Wu; shanhewu@gmail.com

Received 21 June 2014; Accepted 5 August 2014; Published 19 August 2014

Academic Editor: Tohru Ozawa

Copyright (c) 2014 Qiliang Huang et al. This is an open access article distributed under the Creative Commons Attribution License, which permits unrestricted use, distribution, and reproduction in any medium, provided the original work is properly cited.

By the use of the way of real analysis, we estimate the weight functions and give some new Hilbert-type integral inequalities in the whole plane with nonhomogeneous kernels and multiparameters. The constant factors related to the hypergeometric function and the beta function are proved to be the best possible. We also consider the equivalent forms, the reverses, and some particular cases in the homogeneous kernels.

\section{Introduction}

If $f(x), g(y) \geq 0,0<\int_{0}^{\infty} f^{2}(x) d x<\infty$, and $0<$ $\int_{0}^{\infty} g^{2}(y) d y<\infty$, then we have (cf. [1])

$$
\begin{aligned}
& \iint_{0}^{\infty} \frac{f(x) g(y)}{x+y} d x d y \\
& \quad<\pi\left(\int_{0}^{\infty} f^{2}(x) d x \int_{0}^{\infty} g^{2}(y) d y\right)^{1 / 2},
\end{aligned}
$$

where the constant factor $\pi$ is the best possible. Inequality (1) is well known as Hilbert's integral inequality, which is important in analysis and its applications (cf. [1, 2]). In recent years, by using the way of weight functions, a number of extensions of (1) were given by Yang (cf. [3]). Noticing that inequality (1) is with a homogenous kernel of degree -1 , a survey of the study of Hilbert-type inequalities with the homogeneous kernels of degree negative numbers and some parameters was given by [4] in 2009. Recently, some inequalities with the homogenous kernels and nonhomogenous kernels have been studied (cf. [5-12]). All of the above integral inequalities are built in the quarter plane of the first quadrant.
In 2007, Yang [13] first gave a Hilbert-type integral inequality with the nonhomogeneous kernel in the whole plane as follows:

$$
\begin{aligned}
& \iint_{-\infty}^{\infty} \frac{f(x) g(y)}{\left(1+e^{x+y}\right)^{\lambda}} d x d y \\
& \quad<B\left(\frac{\lambda}{2}, \frac{\lambda}{2}\right)\left(\int_{-\infty}^{\infty} e^{-\lambda x} f^{2}(x) d x \int_{-\infty}^{\infty} e^{-\lambda y} g^{2}(y) d y\right)^{1 / 2},
\end{aligned}
$$

where the constant factor $B(\lambda / 2, \lambda / 2)(\lambda>0)$ is the best possible. If $0<\lambda<1, p>1$, and $(1 / p)+(1 / q)=1$, Yang [14] gave another new Hilbert-type integral inequality in the whole plane in 2008 as follows:

$$
\begin{aligned}
\iint_{-\infty}^{\infty} \frac{1}{|1+x y|^{\lambda}} f(x) g(y) d x d y \\
<k_{\lambda}\left\{\int_{-\infty}^{\infty}|x|^{p(1-(\lambda / 2))-1} f^{p}(x) d x\right\}^{1 / p} \\
\quad \times\left\{\int_{-\infty}^{\infty}|y|^{q(1-(\lambda / 2))-1} g^{q}(y) d y\right\}^{1 / q}
\end{aligned}
$$


where the constant factor

$$
k_{\lambda}:=B\left(\frac{\lambda}{2}, \frac{\lambda}{2}\right)+2 B\left(1-\lambda, \frac{\lambda}{2}\right)
$$

is the best possible. He et al. [15-20] also provided some Hilbert-type integral inequalities in the whole plane by using some new methods and techniques.

In this paper, by the use of the way of real analysis, we estimate the weight functions and give some new Hilberttype integral inequalities in the whole plane with nonhomogeneous kernels and multiparameters, which are extensions of (3). The constant factors related to the hypergeometric function and the beta function are proved to be the best possible. We also consider the equivalent forms, the reverses, and some particular inequalities with the homogeneous kernels.

\section{Some Lemmas}

Assuming that $\alpha>0$, we have $\Gamma(\alpha)=\int_{0}^{\infty} x^{\alpha-1} e^{-x} d x$, where $\Gamma(\alpha)$ is the $\Gamma$ function (cf. [21]). For $\beta>-1, \eta>0$, setting $v=-\eta \ln t$, we find the following expression:

$$
\int_{0}^{1}(-\ln t)^{\beta} t^{\eta-1} d t=\frac{1}{\eta^{\beta+1}}=\int_{0}^{\infty} v^{(\beta+1)-1} e^{-v} d v=\frac{\Gamma(\beta+1)}{\eta^{\beta+1}} .
$$

Lemma 1. If $\beta>-1, \min \{\mu, \sigma\}>-\alpha, \mu+\sigma=\lambda<1+\beta$, and $\delta \in\{-1,1\}$, define the weight functions $\omega_{\delta}(\sigma, y)$ and $\omega_{\delta}(\sigma, x)$ as follows:

$$
\begin{aligned}
\omega_{\delta}(\sigma, y):=\int_{-\infty}^{\infty} & \frac{\left.|\ln | x^{\delta} y\right|^{\beta}}{\left|1+x^{\delta} y\right|^{\lambda}} \\
& \times\left(\frac{\min \left\{1,\left|x^{\delta} y\right|\right\}}{\max \left\{1,\left|x^{\delta} y\right|\right\}}\right)^{\alpha} \frac{|y|^{\sigma}}{|x|^{1-\delta \sigma}} d x, \\
\omega_{\delta}(\sigma, x):=\int_{-\infty}^{\infty} \frac{\left.|\ln | x^{\delta} y\right|^{\beta}}{\left|1+x^{\delta} y\right|^{\lambda}} & \\
& \times\left(\frac{\min \left\{1,\left|x^{\delta} y\right|\right\}}{\max \left\{1,\left|x^{\delta} y\right|\right\}}\right)^{\alpha} \frac{|x|^{\delta \sigma}}{|y|^{1-\sigma}} d y .
\end{aligned}
$$

Then, for $y, x \in(-\infty, 0) \cup(0,+\infty)$, we have

$$
\begin{aligned}
\omega_{\delta}(\sigma, y)= & \omega_{\delta}(\sigma, x)=K_{\beta}(\sigma) \\
:= & 2 \Gamma(\beta+1) \sum_{k=0}^{\infty}\left(\begin{array}{c}
\lambda+2 k-1 \\
2 k
\end{array}\right) \\
& \times\left[\frac{1}{(2 k+\sigma+\alpha)^{\beta+1}}\right. \\
& \left.+\frac{1}{(2 k+\mu+\alpha)^{\beta+1}}\right] \in \mathbf{R}_{+} .
\end{aligned}
$$

Proof. (i) For $\delta=1$, setting $u=x y$, we find, for $y \in(-\infty, 0) \cup$ $(0,+\infty)$,

$$
\begin{aligned}
\omega_{1}(\sigma, y) & =\int_{-\infty}^{\infty} \frac{|\ln | u||^{\beta}}{|1+u|^{\lambda}}\left(\frac{\min \{1,|u|\}}{\max \{1,|u|\}}\right)^{\alpha}|u|^{\sigma-1} d u \\
= & \int_{0}^{\infty} \frac{|\ln u|^{\beta}(\min \{1, u\})^{\alpha} u^{\sigma-1}}{(1+u)^{\lambda}(\max \{1, u\})^{\alpha}} d u \\
& +\int_{-\infty}^{0} \frac{|\ln (-u)|^{\beta}(\min \{1,-u\})^{\alpha}}{|1+u|^{\lambda}(\max \{1,-u\})^{\alpha}}(-u)^{\sigma-1} d u \\
= & \int_{0}^{\infty}\left[\frac{|\ln u|^{\beta}(\min \{1, u\})^{\alpha} u^{\sigma-1}}{(1+u)^{\lambda}(\max \{1, u\})^{\alpha}}\right. \\
& \left.+\frac{|\ln u|^{\beta}(\min \{1, u\})^{\alpha} u^{\sigma-1}}{|1-u|^{\lambda}(\max \{1, u\})^{\alpha}}\right] d u \\
& +\int_{0}^{1}\left[\frac{(-\ln u)^{\beta} u^{\sigma+\alpha-1}}{(1+u)^{\lambda}}+\frac{(-\ln u)^{\beta} u^{\sigma+\alpha-1}}{(1-u)^{\lambda}}\right] d u \\
& \int_{1}^{\infty}\left[\frac{(\ln u)^{\beta} u^{\sigma-\alpha-1}}{(1+u)^{\lambda}}+\frac{(\ln u)^{\beta} u^{\sigma-\alpha-1}}{(u-1)^{\lambda}}\right] d u .
\end{aligned}
$$

By Lebesgue term-by-term integration theorem (cf. [22]), in view of (8) and (5), we find

$$
\left.\begin{array}{rl}
\omega_{1}(\sigma, y)= & \int_{0}^{1}\left[\frac{1}{(1+u)^{\lambda}}+\frac{1}{(1-u)^{\lambda}}\right] \\
& \times(-\ln u)^{\beta}\left(u^{\sigma+\alpha-1}+u^{\mu+\alpha-1}\right) d u \\
= & \int_{0}^{1} \sum_{k=0}^{\infty}\left(\begin{array}{c}
-\lambda \\
k
\end{array}\right)\left[u^{k}+(-u)^{k}\right] \\
& \times(-\ln u)^{\beta}\left(u^{\sigma+\alpha-1}+u^{\mu+\alpha-1}\right) d u \\
= & 2 \int_{0}^{1} \sum_{k=0}^{\infty}\left(\begin{array}{c}
\lambda+2 k-1 \\
2 k
\end{array}\right) \\
& \times(-\ln u)^{\beta}\left(u^{2 k+\sigma+\alpha-1}+u^{2 k+\mu+\alpha-1}\right) d u \\
= & 2 \sum_{k=0}^{\infty}\left(\begin{array}{c}
\lambda+2 k-1 \\
2 k
\end{array}\right) \\
= & \times \int_{0}^{1}(-\ln u)^{\beta}\left(u^{2 k+\sigma+\alpha-1}+u^{2 k+\mu+\alpha-1}\right) d u \\
(2 k+\sigma) \sum_{k=0}^{\infty}(\lambda+2 k-1) & 1 k \\
(2 k+\mu+\alpha)^{\beta+1}
\end{array}\right] .
$$


(ii) For $\delta=-1$, setting $y / x$, we still can obtain $\omega_{-1}(\sigma, y)=$ $K_{\beta}(\sigma)$. Setting $u=x^{\delta} y$, we find

$$
\omega_{\delta}(\sigma, x)=\int_{-\infty}^{\infty} \frac{|\ln | u||^{\beta}}{|1+u|^{\lambda}}\left(\frac{\min \{1,|u|\}}{\max \{1,|u|\}}\right)^{\alpha}|u|^{\sigma-1} d u=K_{\beta}(\sigma) .
$$

Since, for $\beta>-1,0<\theta_{0}<\min \{\mu+\alpha, \sigma+\alpha\}$,

$$
\begin{aligned}
& \left(\frac{-\ln u}{1-u}\right)^{\beta} u^{\theta_{0}} \longrightarrow 0 \quad\left(u \longrightarrow 0^{+}\right), \\
& \left(\frac{-\ln u}{1-u}\right)^{\beta} u^{\theta_{0}} \longrightarrow 1 \quad\left(u \longrightarrow 1^{-}\right),
\end{aligned}
$$

there exists a positive number $L$, such that $((-\ln u) /(1-$ $u))^{\beta} u^{\theta_{0}} \leq L(u \in(0,1])$; then, by (9), it follows that

$$
\begin{gathered}
0<K_{\beta}(\sigma) \leq 2 L \int_{0}^{1} \frac{u^{\sigma+\alpha-\theta_{0}-1}+u^{\mu+\alpha-\theta_{0}-1}}{(1-u)^{\lambda-\beta}} d u \\
=2 L\left[B\left(1-\lambda+\beta, \sigma+\alpha-\theta_{0}\right)\right. \\
\left.+B\left(1-\lambda+\beta, \mu+\alpha-\theta_{0}\right)\right]<\infty,
\end{gathered}
$$

and then $K_{\beta}(\sigma) \in \mathbf{R}_{+}$. Hence we have (7).

Remark 2. We have the following formula of the hypergeometric function $F$ (cf. [21]). If $\operatorname{Re}(\gamma)>\operatorname{Re}(\theta)>0, \mid \arg (1-$ $z) \mid<\pi$, then

$$
\begin{aligned}
F(\alpha, \theta, \gamma, z):= & \frac{\Gamma(\gamma)}{\Gamma(\theta) \Gamma(\gamma-\theta)} \\
& \times \int_{0}^{1} t^{\theta-1}(1-t)^{\gamma-\theta-1}(1-z t)^{-\alpha} d t .
\end{aligned}
$$

In particular, for $z=-1, \gamma=\theta+1(\theta>0)$, it follows that

$$
\int_{0}^{1} t^{\theta-1}(1+t)^{-\alpha} d t=\frac{1}{\theta} F(\alpha, \theta, 1+\theta,-1) \in \mathbf{R}_{+} .
$$

In (9), for $\beta=0(\lambda<1)$, in view of (14), we have

$$
\begin{aligned}
K_{0}(\sigma)= & \int_{0}^{1}\left[\frac{1}{(1+u)^{\lambda}}+\frac{1}{(1-u)^{\lambda}}\right] \\
& \times\left(u^{\sigma+\alpha-1}+u^{\mu+\alpha-1}\right) d u \\
= & \frac{1}{\sigma+\alpha} F(\lambda, \sigma+\alpha, 1+\sigma+\alpha,-1) \\
& +\frac{1}{\mu+\alpha} F(\lambda, \mu+\alpha, 1+\mu+\alpha,-1) \\
& +B(1-\lambda, \sigma+\alpha)+B(1-\lambda, \mu+\alpha) \in \mathbf{R}_{+} .
\end{aligned}
$$

Lemma 3. If $p>1,(1 / p)+(1 / q)=1, \beta>-1, \min \{\mu, \sigma\}>$ $-\alpha, \mu+\sigma=\lambda<1+\beta, \delta \in\{-1,1\}, K_{\beta}(\sigma)$ is indicated by (7), and $f(x)$ is a nonnegative measurable function in $(-\infty, \infty)$, then one has

$$
\begin{aligned}
J:=\int_{-\infty}^{\infty}|y|^{p \sigma-1} & {\left[\int_{-\infty}^{\infty} \frac{|\ln | x^{\delta} y||^{\beta}}{\left|1+x^{\delta} y\right|^{\lambda}}\right.} \\
& \left.\times\left(\frac{\min \left\{1,\left|x^{\delta} y\right|\right\}}{\max \left\{1,\left|x^{\delta} y\right|\right\}}\right)^{\alpha} f(x) d x\right]^{p} d y \\
\leq & K_{\beta}^{p}(\sigma) \int_{-\infty}^{\infty}|x|^{p(1-\delta \sigma)-1} f^{p}(x) d x .
\end{aligned}
$$

Proof. By Hölder's inequality (cf. [23]), we have

$$
\begin{aligned}
& {\left[\int_{-\infty}^{\infty} \frac{\left.|\ln | x^{\delta} y\right|^{\beta}}{\left|1+x^{\delta} y\right|^{\lambda}}\left(\frac{\min \left\{1,\left|x^{\delta} y\right|\right\}}{\max \left\{1,\left|x^{\delta} y\right|\right\}}\right)^{\alpha} f(x)\right]^{p}} \\
& =\left[\int_{-\infty}^{\infty} \frac{\left.|\ln | x^{\delta} y\right|^{\beta}}{\left|1+x^{\delta} y\right|^{\lambda}}\left(\frac{\min \left\{1,\left|x^{\delta} y\right|\right\}}{\max \left\{1,\left|x^{\delta} y\right|\right\}}\right)^{\alpha}\right. \\
& \left.\times\left(\frac{|x|^{(1-\delta \sigma) / q}}{|y|^{(1-\sigma) / q}} f(x)\right)\left(\frac{|y|^{(1-\sigma) / q}}{|x|^{(1-\delta \sigma) / q}}\right) d x\right]^{p} \\
& \leq \int_{-\infty}^{\infty} \frac{|\ln | x^{\delta} y||^{\beta}}{\left|1+x^{\delta} y\right|^{\lambda}}\left(\frac{\min \left\{1,\left|x^{\delta} y\right|\right\}}{\max \left\{1,\left|x^{\delta} y\right|\right\}}\right)^{\alpha} \\
& \times \frac{|x|^{(1-\delta \sigma)(p-1)}}{|y|^{1-\sigma}} f^{p}(x) d x \\
& \times\left(\int_{-\infty}^{\infty} \frac{\left.|\ln | x^{\delta} y\right|^{\beta}}{\left|1+x^{\delta} y\right|^{\lambda}}\left(\frac{\min \left\{1,\left|x^{\delta} y\right|\right\}}{\max \left\{1,\left|x^{\delta} y\right|\right\}}\right)^{\alpha}\right. \\
& \left.\times \frac{|y|^{(1-\sigma)(q-1)}}{|x|^{1-\delta \sigma}} d x\right)^{p-1} \\
& =\frac{\left(\omega_{\delta}(\sigma, y)\right)^{p-1}}{|y|^{p \sigma-1}} \\
& \times \int_{-\infty}^{\infty} \frac{\left.|\ln | x^{\delta} y\right|^{\beta}}{\left|1+x^{\delta} y\right|^{\lambda}}\left(\frac{\min \left\{1,\left|x^{\delta} y\right|\right\}}{\max \left\{1,\left|x^{\delta} y\right|\right\}}\right)^{\alpha} \\
& \times \frac{|x|^{(1-\delta \sigma)(p-1)}}{|y|^{1-\sigma}} f^{p}(x) d x .
\end{aligned}
$$


Then, by (7) and Fubini theorem (cf. [22]), it follows that

$$
\begin{aligned}
J \leq K_{\beta}^{p-1}(\sigma) \int_{-\infty}^{\infty}\left[\int_{-\infty}^{\infty} \frac{|\ln | x^{\delta} y||^{\beta}}{\left|1+x^{\delta} y\right|^{\lambda}}\left(\frac{\min \left\{1,\left|x^{\delta} y\right|\right\}}{\max \left\{1,\left|x^{\delta} y\right|\right\}}\right)^{\alpha}\right. \\
\left.\times \frac{|x|^{(1-\delta \sigma)(p-1)}}{|y|^{1-\sigma}} f^{p}(x) d x\right] d y \\
=K_{\beta}^{p-1}(\sigma) \int_{-\infty}^{\infty} \omega_{\delta}(\sigma, x)|x|^{p(1-\delta \sigma)-1} f^{p}(x) d x .
\end{aligned}
$$

Hence, in view of (7), inequality (16) follows.

\section{Main Results and Applications}

Theorem 4. If $p>1,(1 / p)+(1 / q)=1, \beta>-1, \min \{\mu, \sigma\}>$ $-\alpha, \mu+\sigma=\lambda<1+\beta$, and $\delta \in\{-1,1\}, f(x), g(y) \geq$ 0 , satisfying $0<\int_{-\infty}^{\infty}|x|^{p(1-\delta \sigma)-1} f^{p}(x) d x<\infty$ and $0<$ $\int_{-\infty}^{\infty}|y|^{q(1-\sigma)-1} g^{q}(y) d y<\infty$, then one has

$$
\begin{aligned}
& I:=\iint_{-\infty}^{\infty} \frac{\left.|\ln | x^{\delta} y\right|^{\beta}}{\left|1+x^{\delta} y\right|^{\lambda}}\left(\frac{\min \left\{1,\left|x^{\delta} y\right|\right\}}{\max \left\{1,\left|x^{\delta} y\right|\right\}}\right)^{\alpha} \\
& \times f(x) g(y) d x d y \\
& <K_{\beta}(\sigma)\left\{\int_{-\infty}^{\infty}|x|^{p(1-\delta \sigma)-1} f^{p}(x) d x\right\}^{1 / p} \\
& \times\left\{\int_{-\infty}^{\infty}|y|^{q(1-\sigma)-1} g^{q}(y) d y\right\}^{1 / q}, \\
& J:=\int_{-\infty}^{\infty}|y|^{p \sigma-1}\left[\int_{-\infty}^{\infty} \frac{\left.|\ln | x^{\delta} y\right|^{\beta}}{\left|1+x^{\delta} y\right|^{\lambda}}\right. \\
& \left.\times\left(\frac{\min \left\{1,\left|x^{\delta} y\right|\right\}}{\max \left\{1,\left|x^{\delta} y\right|\right\}}\right)^{\alpha} f(x) d x\right]^{p} d y \\
& <K_{\beta}^{p}(\sigma) \int_{-\infty}^{\infty}|x|^{p(1-\delta \sigma)-1} f^{p}(x) d x
\end{aligned}
$$

where the constant factors $K_{\beta}(\sigma)$ and $K_{\beta}^{p}(\sigma)$ are the best possible and $K_{\beta}(\sigma)$ is defined by (7). Inequalities (19) and (20) are equivalent.

In particular, for $\delta=1$, we have the following equivalent inequalities:

$$
\begin{aligned}
\iint_{-\infty}^{\infty} & \frac{|\ln | x y||^{\beta}}{|1+x y|^{\lambda}}\left(\frac{\min \{1,|x y|\}}{\max \{1,|x y|\}}\right)^{\alpha} \\
& \times f(x) g(y) d x d y
\end{aligned}
$$

$$
\begin{gathered}
<K_{\beta}(\sigma)\left\{\int_{-\infty}^{\infty}|x|^{p(1-\sigma)-1} f^{p}(x) d x\right\}^{1 / p} \\
\times\left\{\int_{-\infty}^{\infty}|y|^{q(1-\sigma)-1} g^{q}(y) d y\right\}^{1 / q}, \\
\int_{-\infty}^{\infty}|y|^{p \sigma-1}\left[\int_{-\infty}^{\infty} \frac{\left.|\ln | x y\right|^{\beta}}{|1+x y|^{\lambda}}\left(\frac{\min \{1,|x y|\}}{\max \{1,|x y|\}}\right)^{\alpha}\right. \\
<K_{\beta}^{p}(\sigma) \int_{-\infty}^{\infty}|x|^{p(1-\sigma)-1} f^{p}(x) d x .
\end{gathered}
$$

Proof. If (17) takes the form of equality for a $y \in(-\infty, 0) \cup$ $(0, \infty)$, then there exist constants $A$ and $B$, such that they are not all zero, and

$$
\begin{aligned}
& A \frac{|x|^{(1-\delta \sigma)(p-1)}}{|y|^{1-\sigma}} f^{p}(x) \\
& \quad=B \frac{|y|^{(1-\sigma)(q-1)}}{|x|^{1-\delta \sigma}} \text { a.e. in }(-\infty, \infty) .
\end{aligned}
$$

We suppose that $A \neq 0$ (otherwise $B=A=0$ ). Then it follows that

$$
|x|^{p(1-\delta \sigma)-1} f^{p}(x)=|y|^{q(1-\sigma)} \frac{B}{A|x|} \text { a.e. in }(-\infty, \infty),
$$

which contradicts the fact that $0<\int_{-\infty}^{\infty}|x|^{p(1-\delta \sigma)-1} f^{p}(x) d x<$ $\infty$. Hence (17) takes the form of strict inequality and so does (16). Then we have (20). By Hölder's inequality (cf. [23]), we find

$$
\begin{aligned}
I= & \int_{-\infty}^{\infty}\left[|y|^{\sigma-(1 / p)} \int_{-\infty}^{\infty} \frac{\left.|\ln | x^{\delta} y\right|^{\beta}}{\left|1+x^{\delta} y\right|^{\lambda}}\right. \\
& \left.\times\left(\frac{\min \left\{1,\left|x^{\delta} y\right|\right\}}{\max \left\{1,\left|x^{\delta} y\right|\right\}}\right)^{\alpha} f(x) d x\right] \\
& \times\left(|y|^{(1 / p)-\sigma} g(y) d y\right) \\
\leq & J^{1 / p}\left\{\int_{-\infty}^{\infty}|y|^{q(1-\sigma)-1} g^{q}(y) d y\right\}^{1 / q} .
\end{aligned}
$$

By (20), we have (19). On the other hand, suppose that (19) is valid. We set

$$
g(y):=|y|^{p \sigma-1}\left[\int_{-\infty}^{\infty} \frac{|\ln | x^{\delta} y||^{\beta}}{\left|1+x^{\delta} y\right|^{\lambda}}\right.
$$

$$
\left.\times\left(\frac{\min \left\{1,\left|x^{\delta} y\right|\right\}}{\max \left\{1,\left|x^{\delta} y\right|\right\}}\right)^{\alpha} f(x) d x\right]^{p-1},
$$


and find $J=\int_{-\infty}^{\infty}|y|^{q(1-\sigma)-1} g^{q}(y) d y$. By (16), we have $J<\infty$. If $J=0$, then (20) is obviously value; if $0<J<\infty$, then, by (19), we obtain

$$
\begin{aligned}
0< & \int_{-\infty}^{\infty}|y|^{q(1-\sigma)-1} g^{q}(y) d y=J=I \\
< & K_{\beta}(\sigma)\left\{\int_{-\infty}^{\infty}|x|^{p(1-\delta \sigma)-1} f^{p}(x) d x\right\}^{1 / p} \\
& \times\left\{\int_{-\infty}^{\infty}|y|^{q(1-\sigma)-1} g^{q}(y) d y\right\}^{1 / q}<\infty \\
J^{1 / p}= & \left\{\int_{-\infty}^{\infty}|y|^{q(1-\sigma)-1} g^{q}(y) d y\right\}^{1 / p} \\
< & K_{\beta}(\sigma)\left\{\int_{-\infty}^{\infty}|x|^{p(1-\delta \sigma)-1} f^{p}(x) d x\right\}^{1 / p} .
\end{aligned}
$$

Hence we have (20), which is equivalent to (19). We indicate two sets $E_{\delta}:=\left\{x \in \mathbf{R} ;|x|^{\delta} \geq 1\right\}$ and $E_{\delta}^{+}:=E_{\delta} \cap \mathbf{R}_{+}=\{x \in$ $\left.\mathbf{R}_{+} ; x^{\delta} \geq 1\right\}$. For $\varepsilon>0$, we define two functions $\tilde{f}(x), \tilde{g}(y)$ as follows:

$$
\begin{aligned}
& \tilde{f}(x):= \begin{cases}|x|^{\delta(\sigma-(2 \varepsilon / p))-1}, & x \in E_{\delta}, \\
0, & x \in \mathbf{R} \backslash E_{\delta},\end{cases} \\
& \tilde{g}(y):= \begin{cases}0, & y \in(-\infty,-1) \cup(1, \infty), \\
|y|^{\sigma+(2 \varepsilon / q)-1}, & y \in[-1,1] .\end{cases}
\end{aligned}
$$

Then we obtain

$$
\begin{aligned}
\widetilde{L}:= & \left\{\int_{-\infty}^{\infty}|x|^{p(1-\delta \sigma)-1} \widetilde{f}^{p}(x) d x\right\}^{1 / p} \\
& \times\left\{\int_{-\infty}^{\infty}|y|^{q(1-\sigma)-1} \widetilde{g}^{q}(y) d y\right\}^{1 / q} \\
= & 2\left\{\int_{E_{\delta}^{+}} x^{-2 \delta \varepsilon-1} d x\right\}^{1 / p} \\
& \times\left\{\int_{0}^{1} y^{2 \varepsilon-1} d y\right\}^{1 / q}=\frac{1}{\varepsilon} .
\end{aligned}
$$

Since, for $Y=-y$, we find

$$
\begin{aligned}
h(x):=\int_{-1}^{1} & \frac{\left.|\ln | x^{\delta} y\right|^{\beta}}{\left|1+x^{\delta} y\right|^{\lambda}}\left(\frac{\min \left\{1,\left|x^{\delta} y\right|\right\}}{\max \left\{1,\left|x^{\delta} y\right|\right\}}\right)^{\alpha} \\
& \times|y|^{\sigma+(2 \varepsilon / q)-1} d y \\
=\int_{-1}^{1} & \frac{\left.|\ln |(-x)^{\delta} Y\right|^{\beta}}{\left|1+(-x)^{\delta} Y\right|^{\lambda}}\left(\frac{\min \left\{1,\left|(-x)^{\delta} Y\right|\right\}}{\max \left\{1,\left|(-x)^{\delta} Y\right|\right\}}\right)^{\alpha} \\
& \times|Y|^{\sigma+(2 \varepsilon / q)-1} d Y=h(-x),
\end{aligned}
$$

and $h(x)$ is an even function, then it follows that

$$
\begin{aligned}
\widetilde{I}:=\iint_{-\infty}^{\infty} \frac{\left.|\ln | x^{\delta} y\right|^{\beta}}{\left|1+x^{\delta} y\right|^{\lambda}}\left(\frac{\min \left\{1,\left|x^{\delta} y\right|\right\}}{\max \left\{1,\left|x^{\delta} y\right|\right\}}\right)^{\alpha} \\
\quad \times \tilde{f}(x) \tilde{g}(y) d x d y \\
=\int_{E_{\delta}}|x|^{\delta(\sigma-(2 \varepsilon / p))-1} h(x) d x \\
=2 \int_{E_{\delta}^{+}} x^{\delta(\sigma-(2 \varepsilon / p))-1} h(x) d x \\
\stackrel{u=x^{\delta} y}{=} 2 \int_{E_{\delta}^{+}} x^{-2 \delta \varepsilon-1} \\
\quad \times\left[\int_{-x^{\delta}}^{x^{\delta}} \frac{|\ln | u||^{\beta}(\min \{1,|u|\})^{\alpha}|u|^{\sigma+(2 \varepsilon / q)-1}}{|1+u|^{\lambda}(\max \{1,|u|\})^{\alpha}} d u\right] d x .
\end{aligned}
$$

Setting $v=x^{\delta}$ in the above integral, by Fubini theorem (cf. [22]), we find

$$
\begin{aligned}
& \widetilde{I} \stackrel{v=x^{\delta}}{=} 2 \int_{1}^{\infty} v^{-2 \varepsilon-1} \\
& \times\left[\int_{-v}^{v} \frac{|\ln | u||^{\beta}(\min \{1,|u|\})^{\alpha}|u|^{\sigma+(2 \varepsilon / q)-1}}{|1+u|^{\lambda}(\max \{1,|u|\})^{\alpha}} d u\right] d v \\
& =2 \int_{1}^{\infty} v^{-2 \varepsilon-1}\left\{\int _ { 0 } ^ { v } \left[\frac{|\ln u|^{\beta}(\min \{1, u\})^{\alpha}}{|1-u|^{\lambda}(\max \{1, u\})^{\alpha}}\right.\right. \\
& \left.+\frac{|\ln u|^{\beta}(\min \{1, u\})^{\alpha}}{(1+u)^{\lambda}(\max \{1, u\})^{\alpha}}\right] \\
& \left.\times u^{\sigma+(2 \varepsilon / q)-1} d u\right\} d v \\
& =2 \int_{1}^{\infty} v^{-2 \varepsilon-1} \\
& \times\left\{\int_{0}^{1}\left[\frac{(-\ln u)^{\beta}}{(1-u)^{\lambda}}+\frac{(-\ln u)^{\beta}}{(1+u)^{\lambda}}\right] u^{\sigma+\alpha+(2 \varepsilon / q)-1} d u\right\} d v \\
& +2 \int_{1}^{\infty} v^{-2 \varepsilon-1} \\
& \times\left\{\int_{1}^{v}\left[\frac{(\ln u)^{\beta}}{(u-1)^{\lambda}}+\frac{(\ln u)^{\beta}}{(1+u)^{\lambda}}\right] u^{\sigma-\alpha+(2 \varepsilon / q)-1} d u\right\} d v \\
& =\frac{1}{\varepsilon} \int_{0}^{1}\left[\frac{(-\ln u)^{\beta}}{(1-u)^{\lambda}}+\frac{(-\ln u)^{\beta}}{(1+u)^{\lambda}}\right] u^{\sigma+\alpha+(2 \varepsilon / q)-1} d u \\
& +2 \int_{1}^{\infty}\left(\int_{u}^{\infty} v^{-2 \varepsilon-1} d v\right) \\
& \times\left[\frac{(\ln u)^{\beta}}{(u-1)^{\lambda}}+\frac{(\ln u)^{\beta}}{(1+u)^{\lambda}}\right] u^{\sigma-\alpha+(2 \varepsilon / q)-1} d u
\end{aligned}
$$




$$
\begin{aligned}
=\frac{1}{\varepsilon}\left\{\int_{0}^{1}\left[\frac{(-\ln u)^{\beta}}{(1-u)^{\lambda}}+\frac{(-\ln u)^{\beta}}{(1+u)^{\lambda}}\right] u^{\sigma+\alpha+(2 \varepsilon / q)-1} d u\right. \\
\left.+\int_{1}^{\infty}\left[\frac{(\ln u)^{\beta}}{(u-1)^{\lambda}}+\frac{(\ln u)^{\beta}}{(1+u)^{\lambda}}\right] u^{\sigma-\alpha-(2 \varepsilon / p)-1} d u\right\} .
\end{aligned}
$$

If the constant factor $K_{\beta}(\sigma)$ in (19) is not the best possible, then there exists a positive number $k$ with $K_{\beta}(\sigma)<k$, such that (19) is valid when replacing $K_{\beta}(\sigma)$ by $k$. Then we have $\widetilde{I}<k \widetilde{L}$, and

$$
\begin{aligned}
& \int_{0}^{1}\left[\frac{(-\ln u)^{\beta}}{(1-u)^{\lambda}}+\frac{(-\ln u)^{\beta}}{(1+u)^{\lambda}}\right] u^{\sigma+\alpha+(2 \varepsilon / q)-1} d u \\
& \quad+\int_{1}^{\infty}\left[\frac{(\ln u)^{\beta}}{(u-1)^{\lambda}}+\frac{(\ln u)^{\beta}}{(1+u)^{\lambda}}\right] u^{\sigma-\alpha-(2 \varepsilon / p)-1} d u \\
& =\varepsilon \widetilde{I}<\varepsilon k \widetilde{L}=k .
\end{aligned}
$$

By (8) and Fatou lemma (cf. [22]), we have

$$
\begin{aligned}
& K_{\beta}(\sigma)=\int_{0}^{1}\left[\frac{(-\ln u)^{\beta}}{(1-u)^{\lambda}}+\frac{(-\ln u)^{\beta}}{(1+u)^{\lambda}}\right] u^{\sigma+\alpha-1} d u \\
& +\int_{1}^{\infty}\left[\frac{(\ln u)^{\beta}}{(u-1)^{\lambda}}+\frac{(\ln u)^{\beta}}{(1+u)^{\lambda}}\right] u^{\sigma-\alpha-1} d u \\
& =\int_{0}^{1} \lim _{\varepsilon \rightarrow 0^{+}}\left[\frac{(-\ln u)^{\beta}}{(1-u)^{\lambda}}+\frac{(-\ln u)^{\beta}}{(1+u)^{\lambda}}\right] u^{\sigma+\alpha+(2 \varepsilon / q)-1} d u \\
& +\int_{1}^{\infty} \lim _{\varepsilon \rightarrow 0^{+}}\left[\frac{(\ln u)^{\beta}}{(u-1)^{\lambda}}+\frac{(\ln u)^{\beta}}{(1+u)^{\lambda}}\right] u^{\sigma-\alpha-(2 \varepsilon / p)-1} d u \\
& \leq \underline{\lim }_{\varepsilon \rightarrow 0^{+}}\left\{\int_{0}^{1}\left[\frac{(-\ln u)^{\beta}}{(1-u)^{\lambda}}+\frac{(-\ln u)^{\beta}}{(1+u)^{\lambda}}\right] u^{\sigma+\alpha+(2 \varepsilon / q)-1} d u\right. \\
& +\int_{1}^{\infty}\left[\frac{(\ln u)^{\beta}}{(u-1)^{\lambda}}+\frac{(\ln u)^{\beta}}{(1+u)^{\lambda}}\right] \\
& \left.\times u^{\sigma-\alpha-(2 \varepsilon / p)-1} d u\right\} \leq k,
\end{aligned}
$$

which contradicts the fact that $k<K_{\beta}(\sigma)$. Hence the constant factor $K_{\beta}(\sigma)$ in (19) is the best possible. If the constant factor in (20) is not the best possible, then, by (24), we may get a contradiction that the constant factor in (19) is not the best possible.

Theorem 5. As the assumptions of Theorem 4, replacing $p>1$ by $0<p<1$, one has the equivalent reverses of (19) and (20) with the same best constant factors.

Proof. By the reverse Hölder's inequality (cf. [23]), we have the reverses of (16) and (24). It is easy to obtain the reverse of (20). In view of the reverses of (20) and (24), we obtain the reverse of (19). On the other hand, suppose that the reverse of (19) is valid. Setting the same $g(y)$ as (25) in Theorem 4, by the reverse of (16), we have $J>0$. If $J=\infty$, then the reverse of (20) is obviously value; if $J<\infty$, then, by the reverse of (19), we obtain the reverses of (26). Hence we have the reverse of (20), which is equivalent to the reverse of (19). If the constant factor $K_{\beta}(\sigma)$ in the reverse of (19) is not the best possible, then there exists a positive constant $k$, with $k>K_{\beta}(\sigma)$, such that the reverse of (19) is still valid when replacing $K_{\beta}(\sigma)$ by $k$. By the reverse of (32), we have

$$
\begin{aligned}
& \int_{0}^{1}\left[\frac{(-\ln u)^{\beta}}{(1-u)^{\lambda}}+\frac{(-\ln u)^{\beta}}{(1+u)^{\lambda}}\right] u^{\sigma+\alpha+(2 \varepsilon / q)-1} d u \\
& \quad+\int_{1}^{\infty}\left[\frac{(\ln u)^{\beta}}{(u-1)^{\lambda}}+\frac{(\ln u)^{\beta}}{(1+u)^{\lambda}}\right] u^{\sigma-\alpha-(2 \varepsilon / p)-1} d u>k .
\end{aligned}
$$

For $\varepsilon \rightarrow 0^{+}$, by Levi theorem (cf. [22]), we find

$$
\begin{aligned}
& \int_{1}^{\infty}\left[\frac{(\ln u)^{\beta}}{(u-1)^{\lambda}}+\frac{(\ln u)^{\beta}}{(1+u)^{\lambda}}\right] u^{\sigma-\alpha-(2 \varepsilon / p)-1} d u \\
& \longrightarrow \int_{1}^{\infty}\left[\frac{(\ln u)^{\beta}}{(u-1)^{\lambda}}+\frac{(\ln u)^{\beta}}{(1+u)^{\lambda}}\right] u^{\sigma-\alpha-1} d u
\end{aligned}
$$

There exists a constant $\delta_{0}>0$, such that $\sigma-(1 / 2) \delta_{0}>-\alpha$, and then $0<K_{\beta}\left(\sigma-\left(\delta_{0} / 2\right)\right)<\infty$. For $0<\varepsilon<\delta_{0}|q| / 4(q<0)$, since $u^{\sigma+\alpha+(2 \varepsilon / q)-1} \leq u^{\sigma+\alpha-\left(\delta_{0} / 2\right)-1}, u \in(0,1]$, and

$$
\int_{0}^{1}\left[\frac{(-\ln u)^{\beta}}{(1-u)^{\lambda}}+\frac{(-\ln u)^{\beta}}{(1+u)^{\lambda}}\right] u^{\sigma+\alpha-\left(\delta_{0} / 2\right)-1} d u \leq K_{\beta}\left(\sigma-\frac{\delta_{0}}{2}\right)
$$

then, by Lebesgue control convergence theorem (cf. [22]), for $\varepsilon \rightarrow 0^{+}$, we have

$$
\begin{gathered}
\int_{0}^{1}\left[\frac{(-\ln u)^{\beta}}{(1-u)^{\lambda}}+\frac{(-\ln u)^{\beta}}{(1+u)^{\lambda}}\right] u^{\sigma+\alpha+(2 \varepsilon / q)-1} d u \\
\longrightarrow \int_{0}^{1}\left[\frac{(-\ln u)^{\beta}}{(1-u)^{\lambda}}+\frac{(-\ln u)^{\beta}}{(1+u)^{\lambda}}\right] u^{\sigma+\alpha-1} d u .
\end{gathered}
$$

By (34), (35), and (37), for $\varepsilon \rightarrow 0^{+}$, we have $K_{\beta}(\sigma) \geq k$, which contradicts the fact that $k>K_{\beta}(\sigma)$. Hence, the constant factor $K_{\beta}(\sigma)$ in the reverse of (19) is the best possible. If the constant factor in the reverse of (20) is not the best possible, then, by the reverse of (24), we may get a contradiction that the constant factor in the reverse of (19) is not the best possible. 
Remark 6. (i) For $\delta=-1$ in (19) and (20), replacing $|x|^{\lambda} f(x)$ by $f(x)$, we obtain the following equivalent inequalities with a homogeneous kernel and the best possible constant factors:

$$
\begin{aligned}
\iint_{-\infty}^{\infty} \frac{|\ln | y /\left.x\right|^{\beta}}{|x+y|^{\lambda}}\left(\frac{\min \{|x|,|y|\}}{\max \{|x|,|y|\}}\right)^{\alpha} f(x) g(y) d x d y \\
<K_{\beta}(\sigma)\left\{\int_{-\infty}^{\infty}|x|^{p(1-\mu)-1} f^{p}(x) d x\right\}^{1 / p} \\
\quad \times\left\{\int_{-\infty}^{\infty}|y|^{q(1-\sigma)-1} g^{q}(y) d y\right\}^{1 / q}, \\
\int_{-\infty}^{\infty}|y|^{p \sigma-1} \\
\quad \times\left[\int_{-\infty}^{\infty} \frac{|\ln | y /\left.x\right|^{\beta}}{|x+y|^{\lambda}}\left(\frac{\min \{|x|,|y|\}}{\max \{|x|,|y|\}}\right)^{\alpha} f(x) d x\right]^{p} d y \\
<K_{\beta}^{p}(\sigma) \int_{-\infty}^{\infty}|x|^{p(1-\mu)-1} f^{p}(x) d x .
\end{aligned}
$$

(ii) For $\beta=0(\lambda<1)$ in (19) and (20), we obtain the following equivalent inequalities:

$$
\begin{aligned}
& \iint_{-\infty}^{\infty} \frac{\left(\min \left\{1,\left|x^{\delta} y\right|\right\}\right)^{\alpha}}{\left|1+x^{\delta} y\right|^{\lambda}\left(\max \left\{1,\left|x^{\delta} y\right|\right\}\right)^{\alpha}} f(x) g(y) d x d y \\
& <K_{0}(\sigma)\left\{\int_{-\infty}^{\infty}|x|^{p(1-\delta \sigma)-1} f^{p}(x) d x\right\}^{1 / p} \\
& \quad \times\left\{\int_{-\infty}^{\infty}|y|^{q(1-\sigma)-1} g^{q}(y) d y\right\}^{1 / q}, \\
& \int_{-\infty}^{\infty}|y|^{p \sigma-1} \\
& \quad \times\left[\int_{-\infty}^{\infty} \frac{\left(\min \left\{1,\left|x^{\delta} y\right|\right\}\right)^{\alpha}}{\left|1+x^{\delta} y\right|^{\lambda}\left(\max \left\{1,\left|x^{\delta} y\right|\right\}\right)^{\alpha}} f(x) d x\right]^{p} d y \\
& <K_{0}^{p}(\sigma) \int_{-\infty}^{\infty}|x|^{p(1-\delta \sigma)-1} f^{p}(x) d x,
\end{aligned}
$$

where $K_{0}(\sigma)$ is indicated by (15).

(iii) For $\alpha=0, \sigma=\mu=\lambda / 2(0<\lambda<1)$ in (40), we find

$$
\begin{aligned}
K_{0}\left(\frac{\lambda}{2}\right)= & \int_{-\infty}^{\infty} \frac{1}{|1+u|^{\lambda}}|u|^{(\lambda / 2)-1} d u \\
= & \int_{0}^{\infty} \frac{u^{(\lambda / 2)-1} d u}{(1+u)^{\lambda}}+\int_{-\infty}^{-1} \frac{(-u)^{(\lambda / 2)-1} d u}{(-1-u)^{\lambda}} \\
& +\int_{-1}^{0} \frac{(-u)^{(\lambda / 2)-1} d u}{(1+u)^{\lambda}} \\
= & B\left(\frac{\lambda}{2}, \frac{\lambda}{2}\right)+2 \int_{0}^{1} \frac{u^{(\lambda / 2)-1}}{(1-u)^{\lambda}} d u=k_{\lambda},
\end{aligned}
$$

and then (3) follows. Hence, (40) and (19) are extensions of (3).

\section{Conflict of Interests}

The authors declare that there is no conflict of interests regarding the publication of this paper.

\section{Acknowledgments}

This work is supported by the National Natural Science Foundation of China (no. 61370186), 2013 Knowledge Construction Special Foundation Item of Guangdong Institution of Higher Learning College and University (no. 2013KJCX0140), and the Foundation of Scientific Research Project of Fujian Province Education Department of China (no. JK2012049).

\section{References}

[1] G. H. Hardy, J. E. Littlewood, and G. Po'lya, Inequalities, Cambridge University Press, Cambridge, Mass, USA, 1934.

[2] D. S. Mitrinović, J. E. Pečarić, and A. M. Fink, Inequalities Involving Functions and Their Integrals and Derivatives, vol. 53 of Boston, Mass, USA, Kluwer Academic Publishers, 1991.

[3] B. Yang, The Norm of Operator and Hilbert-Type Inequalities, Science Press, Beijing, China, 2009.

[4] B. C. Yang, "A survey of the study of Hilbert-type inequalities with parameters," Advances in Mathematics, vol. 38, no. 3, pp. 257-268, 2009.

[5] B. Yang, "On the norm of an integral operator and applications," Journal of Mathematical Analysis and Applications, vol. 321, no. 1, pp. 182-192, 2006.

[6] I. Brnetic and J. Pecaric, "Generalizations of Hilbert's integral inequality," Mathematical Inequalities and Applications, vol. 7, no. 2, pp. 199-205, 2004.

[7] M. Krnić and J. Pečarić, "General Hilbert's and Hardy's inequalities," Mathematical Inequalities \& Applications, vol. 8, no. 1, pp. 29-51, 2005.

[8] J. Xu, "Hardy-Hilbert's inequalities with two parameters," Advances in Mathematics, vol. 36, no. 2, pp. 189-202, 2007.

[9] B. Yang, "On the norm of a Hilbert's type linear operator and applications," Journal of Mathematical Analysis and Applications, vol. 325, no. 1, pp. 529-541, 2007.

[10] D. M. Xin, "A Hilbert-type integral inequality with a homogeneous kernel of zero degree," Mathematical Theory and Applications, vol. 30, no. 2, pp. 70-74, 2010.

[11] Q. Liu and W. Sun, "A Hilbert-type integral inequality with multiparameters and a nonhomogeneous kernel," Abstract and Applied Analysis, vol. 2014, Article ID 674874, 5 pages, 2014.

[12] L. Debnath and B. Yang, "Recent developments of Hilbert-type discrete and integral inequalities with applications," International Journal of Mathematics and Mathematical Sciences, vol. 2012, Article ID 871845, 29 pages, 2012.

[13] B. Yang, "A new Hilbert's type integral inequality," Soochow Journal of Mathematics, vol. 33, no. 4, pp. 849-859, 2007.

[14] B. C. Yang, "A new Hilbert-type integral inequality with some parameters," Journal of Jilin University (Science Edition), vol. 46, no. 6, pp. 1085-1090, 2008.

[15] B. He and B. C. Yang, "A Hilbert-type integral inequality with a homogeneous kernel of 0-degree and a hypergeometric function," Mathematics in Practice and Theory. Shuxue de Shijian yu Renshi, vol. 40, no. 18, pp. 203-211, 2010. 
[16] Z. Zeng and Z. Xie, "On a new Hilbert-type integral inequality with the homogeneous kernel of degree 0 and the integral in whole plane," Journal of Inequalities and Applications, vol. 2010, Article ID 256796, 9 pages, 2010.

[17] A. Wang and B. Yang, "A new Hilbert-type integral inequality in the whole plane with the non-homogeneous kernel," Journal of Inequalities and Applications, vol. 2011, article 123, 2011.

[18] B. He and B. Yang, "On an inequality concerning a nonhomogeneous kernel and the hypergeometric function," Tamsui Oxford Journal of Information and Mathematical Sciences, vol. 27, no. 1, pp. 75-88, 2011.

[19] Z. Xie, Z. Zeng, and Y. Sun, "A new Hilbert-type inequality with the homogeneous kernel of degree," Advances and Applications in Mathematical Sciences, vol. 12, no. 7, pp. 391-401, 2013.

[20] Z. Zhen, K. R. R. Gandhi, and Z. Xie, "A new Hilbert-type inequality with the homogeneous kernel of degree-2 and with the integral," Bulletin of Mathematical Sciences \& Applications, vol. 3, no. 1, pp. 11-20, 2014.

[21] Z. Wang and D. Guo, Introduction to Special Functions, Science Press, Beijing, China, 1979.

[22] J. Kuang, Introudction to Real Analysis, Hunan Educiton Press, Changsha, China, 1996.

[23] J. Kuang, Applied Inequalities, Shangdong Science and Technology Press, Jinan, China, 2004. 


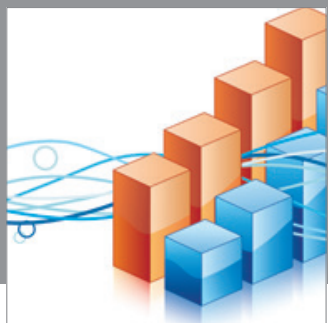

Advances in

Operations Research

mansans

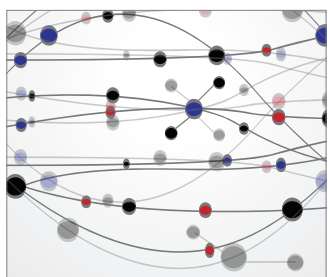

The Scientific World Journal
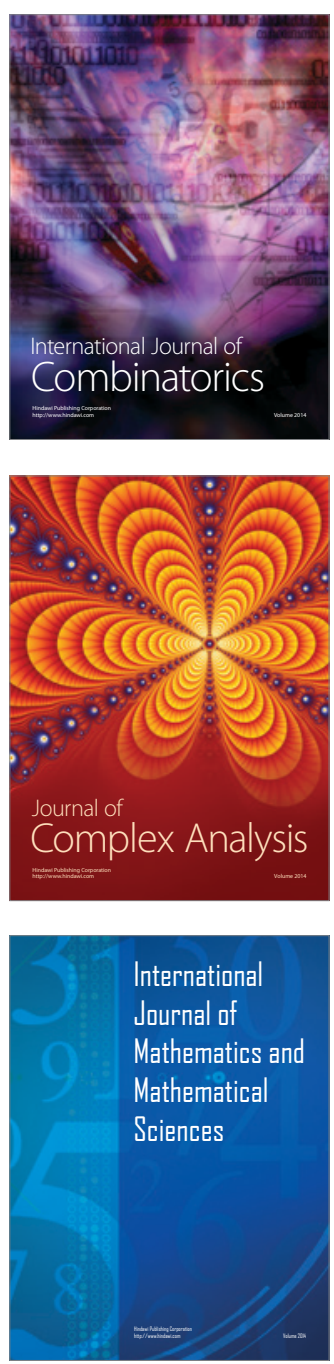
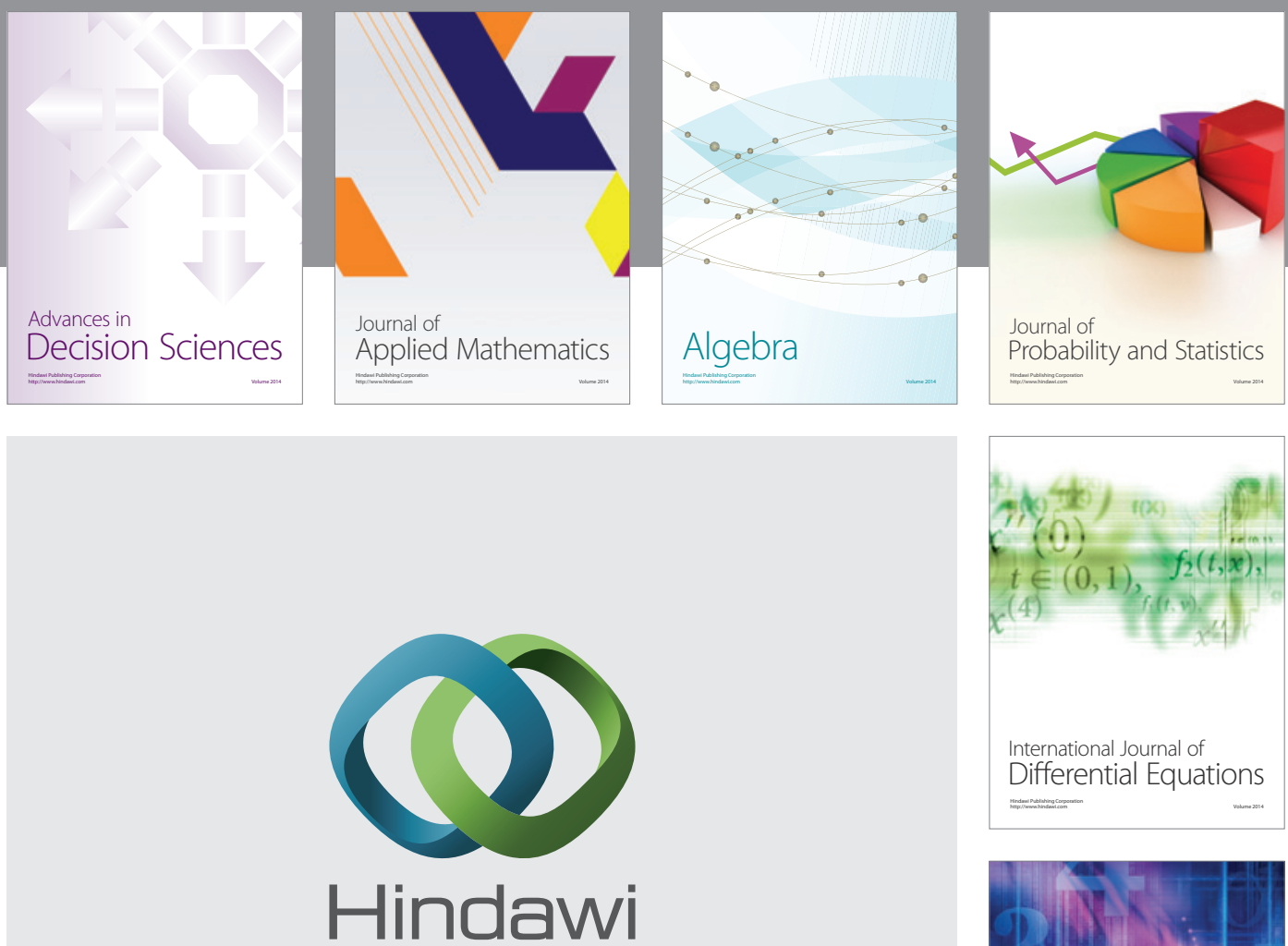

Submit your manuscripts at http://www.hindawi.com
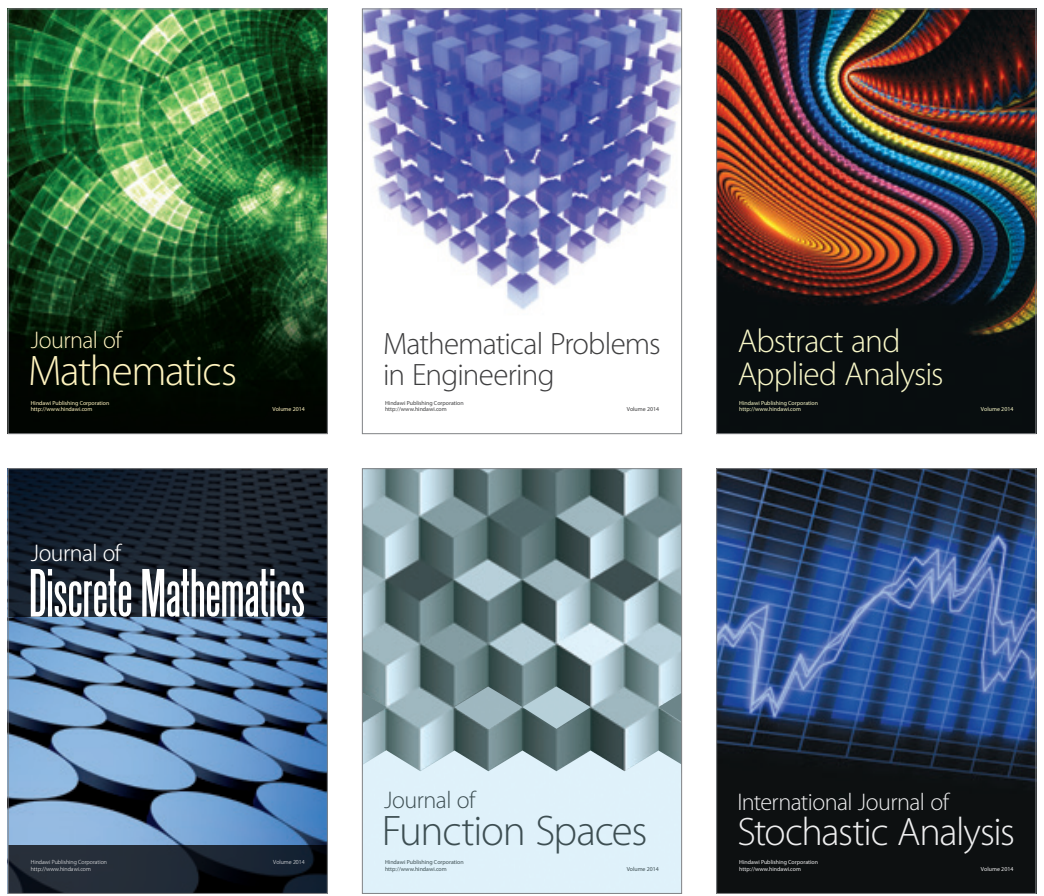

Journal of

Function Spaces

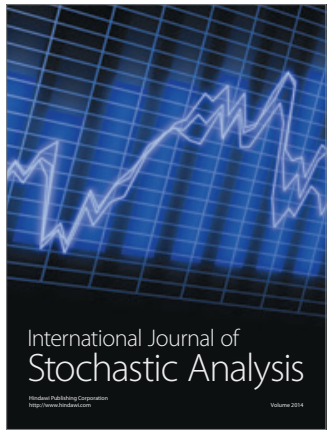

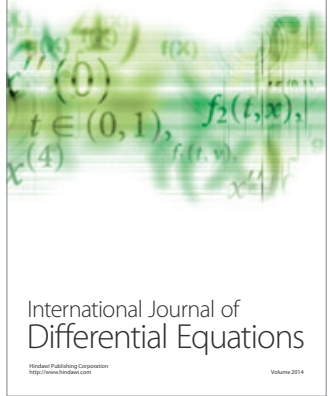
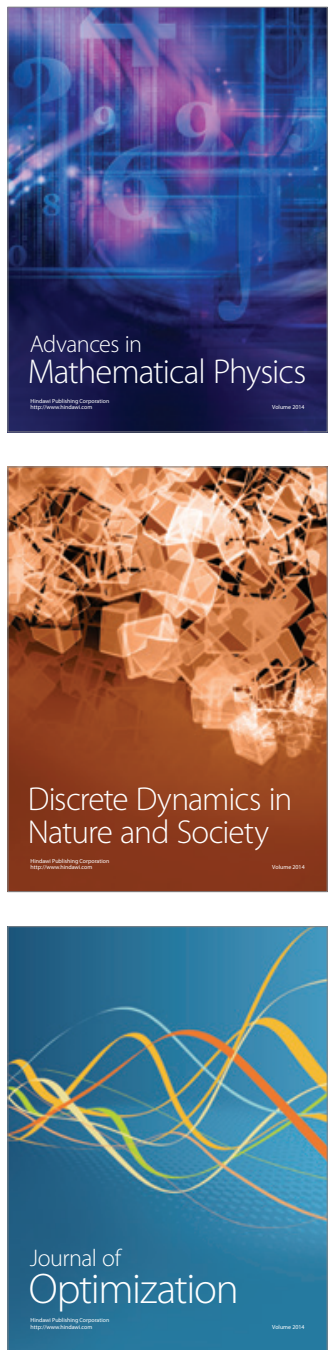